Pneumologe 2019 · 16:67-68

https://doi.org/10.1007/s10405-019-0233-y

(c) Springer Medizin Verlag $\mathrm{GmbH}$, ein Teil von Springer Nature 2019

CrossMark

\author{
J. Behr ${ }^{1,2} \cdot$ E. von Mutius ${ }^{3}$ \\ ${ }^{1}$ Medizinische Klinik u. Poliklinik V (Pneumologie), Ludwig-Maximilians-Universität, Campus Großhadern, \\ München, Deutschland \\ ${ }^{2}$ Asklepios Fachkliniken München-Gauting, Mitglied des Deutschen Zentrums für Lungenforschung, \\ München, Deutschland \\ ${ }^{3}$ Dr. von Haunerschen Kinderspital, Ludwig-Maximilians-Universität, München, Deutschland
}

\title{
Personalisierte Medizin in der Pneumologie
}

übergreifende Pathomechanismen der Karzinogenese erkennen. Darüber hinaus konnte in den Jahren 2017 und 2018 auch der Traum von der Immuntherapie von Tumorerkrankungen verwirklicht werden. Eine Voraussetzung hierfür waren grundlegende Arbeiten zur Immunregulation der Tumorabwehr, für die James P. Allison und Tasuku Honjo den diesjährigen Medizinnobelpreis erhielten. Es zeichnet sich ab, dass die Kombination aus Immuntherapie und klassischer Chemotherapie in Zukunft ein Therapiestandard für viele Patienten mit unterschiedlichen Formen des Lungenkrebses einschließlich kleinzelligem Lungenkarzinom wird. Gerade auf dem Gebiet des Lungenkarzinoms besteht aktuell eine sehr große Dynamik, welche die thorakale Onkologie vor die Herausforderung stellt, individuelle, vorausschauende Therapiestrategien $\mathrm{zu}$ entwickeln.

Der Beitrag von Frau Katrin MilgerKneidinger aus der Medizinischen Klinik und Poliklinik V des Klinikums der Universität München zur pulmonalen $\mathrm{Hy}$ pertonie macht deutlich, dass auf diesem Gebiet der Pneumologie die individualisierte Therapie bereits eine lange Tradition hat. So erlaubte die empirisch entwickelte Vasoreagibilitätstestung bereits frühzeitig Patienten zu identifizieren, die hervorragend auf eine Therapie mit Kalziumantagonisten ansprechen und im positiven Fall eine sehr günstige Langzeitprognose aufweisen, ganz im Gegensatz zu Patienten mit pulmonal arterieller Hypertonie mit fehlender Vasoreagibilität. Aber auch auf diesem Ge- biet ging die Entwicklung weiter und mit der Identifizierung von drei molekularen Signalwegen zur therapeutischen Beeinflussung der Lungengefäßstrombahn steht heute eine Vielzahl unterschiedlicher medikamentöser Therapieansätze zur Verfügung, die eine individuell optimierte Therapiestrategie zur Erreichung definierter Therapieziele erlauben.

Ein weiteres Highlight der personalisierten Medizin stellt der Beitrag von Frau Silke von Koningsbruggen-Rietschel aus der Kinderklinik Köln dar. Ursache der Erkrankung ist eine Mutation des CFTR-Gens (Cystic Fibrosis Transmembran Regulator), ein Membranprotein, das als Chloridkanal dient und bei Funktionsstörung zu einer Sekreteindickung in allen drüsigen Organen einschließlich Pankreas und Lunge führt. Inzwischen sind über 1900 verschiedene Mutationen bekannt und mit der Wahrscheinlichkeit der Geburt eines Kindes mit Mukoviszidose in Europa von 1:2000 ist die Mukoviszidose die häufigste autosomal-rezessive Erbkrankheit und die häufigste letale genetische Erbkrankheit in der hellhäutigen Bevölkerung. Während gentherapeutische Therapieansätze bisher nicht verwirklicht werden konnten, ist es gelungen, klassisch pharmakologisch wirkende Medikamente zu entwickeln, welche zu einer Optimierung der Restaktivität des mutierten CFTR-Gens führen und so sowohl die klinische Symptomatik als auch den Krankheitsverlauf bei bestimmten Genvarianten sehr positiv beeinflussen. Während die Mukoviszidose noch vor 30 Jahren in der Regel vor Erreichen der Geschlechtsreife zum lässt auch gemeinsame tumorentitäts- 
Tod des Patienten führte, ist es durch Einsatz klassischer Therapiestrategien - einschließlich Physiotherapie, Inhalationstherapie und Antibiotika - gelungen, die Lebenserwartung der Patienten auf heute durchschnittlich 40 Jahre zu erhöhen. Die neuen molekularen Therapiestrategien versprechen eine weitere Verbesserung dieser Erfolge.

Der vierte Beitrag dieses Sonderhefts von Frau Nicole Maison aus der Dr. von Haunerschen Kinderklinik der Universität München zeigt das Wissen auf, wie sich die Erkrankung Asthma vom Kind bis zum Erwachsenen entwickelt. Es gibt bislang wenig Kollaboration zwischen pädiatrischer und adulter Pneumologie, weshalb der gemeinsame Kongress von Deutscher Gesellschaft für Pneumologie und Gesellschaft für Pädiatrische Pneumologie auch helfen will, die Brücke zu spannen. Aus der Epidemiologie gibt es aber bereits solche Brücken. Diese werden von etlichen Kohorten gebildet, die Kinder über die Adoleszenz bis ins Erwachsenenalter verfolgt haben. Dabei wurden die Progression und Remission von Beschwerden wie auch die relevanten Einflussfaktoren untersucht. Auch die Entwicklung der Lungenfunktion ist aus diesen Kohorten ablesbar. Es wird dabei zunehmend klar, dass viele Probleme im Erwachsenenalter ihre Wurzeln in der Kindheit haben, ob es sich jetzt um Asthma bronchiale oder die chronische obstruktive Lungenerkrankung (COPD) handelt. Gerade in diesem Bereich scheint eine Kollaboration über Altersgrenzen hinweg von besonderer Bedeutung, wie sie auch im Deutschen Zentrum für Lungenforschung praktiziert wird.

Das Sonderheft personalisierte Medizin in der Pneumologie zeigt exemplarisch an vier Subspezialitäten des Fachgebiets, wie technischer Fortschritt und Innovationen $\mathrm{zu}$ individualisierten Therapiestrategien führen und bisher unerreichbar geglaubte Behandlungserfolge ermöglichen. Die Pneumologie nimmt bezüglich dieser Entwicklung der perso- nalisierten Medizin und Präzisionsmedizin innerhalb der Inneren Medizin eine führende Position ein.
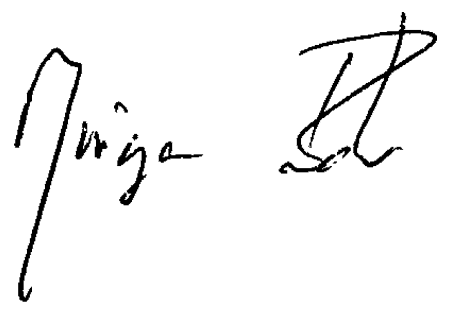

Prof. Dr. Jürgen Behr

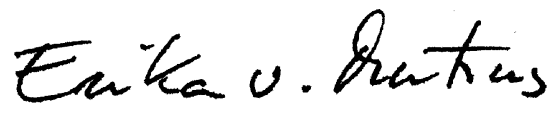

Prof. Dr. Dr. h. c. Erika von Mutius

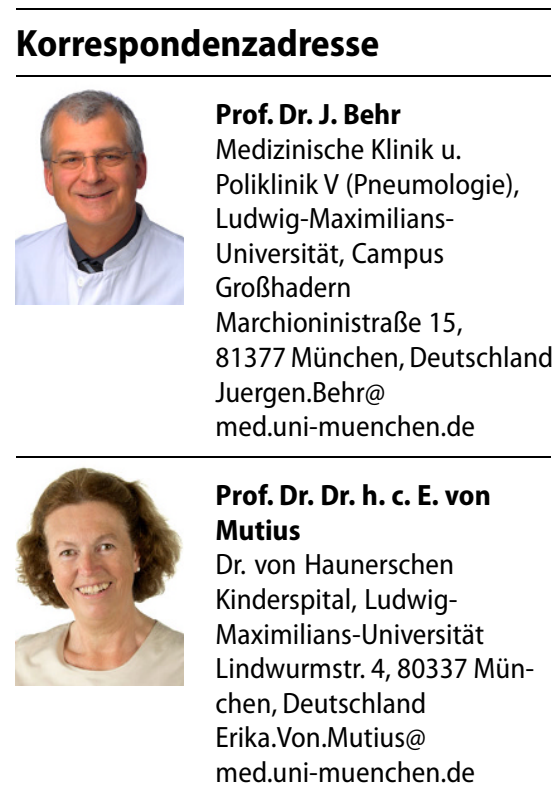

Interessenkonflikt. J. Behr und E. von Mutius geben an, dass kein Interessenkonflikt besteht.

\section{Spezifische Immuntherapie jetzt als Asthmatherapie empfohlen}

Zur Behandlung von Asthma wird die spezifische Immuntherapie (SIT) nun ausdrücklich in der neu aufgelegten Nationalen Versorgungsleitlinie (NVL) Asthma empfohlen. „Für Asthmapatienten ist das fast wie eine Revolution“ beurteilt Prof. Dr. med. Eckard Hamelmann, Vizepräsident der Deutschen Gesellschaft für Allergologie und Klinische Immunologie (DGAKI) und Koautor der Leitlinie diese Neuerung.

Bei Asthma-Patienten soll der behandelnde Arzt im Rahmen der Asthma-Diagnostik jetzt auch eine allergologische Abklärung vornehmen, wenn der Verdacht auf eine Allergie besteht oder wenn eine andere allergische Erkrankung vorliegt.

Für die Therapie legt die neue NVL Asthma fest, dass die spezifische Immuntherapie jetzt ausdrücklich zur Stufentherapie gehört. Sie gilt für alle Stufen als zusätzliche Therapieoption, unabhängig vom Schweregrad. Patienten haben somit ein Anrecht auf Aufklärung über die Möglichkeit einer spezifischen Immuntherapie zur Behandlung ihres Asthmas.

Die spezifische Immuntherapie ist die einzig kausale Behandlungsform für allergisch bedingte Erkrankungen. Neue Studien haben gezeigt, dass die spezifische Immuntherapie bei allergischem Asthma Exazerbationen verringern und die Symptomkontrolle verbessern kann. „In Kinderstudien konnte gezeigt werden, dass die Anwendung von Kortikosteroiden durch die spezifische Immuntherapie reduziert werden kann" betont Prof. Hamelmann.

Kontakt:

Prof. Dr. med. Eckard Hamelmann, Kinderzentrum Bethel, Evangelisches Klinikum Bethel: kinderklinik@evkb.de Prof. Dr. med. Kleine-Tebbe, Pressesprecher DGAKI: kleine-tebbe@allergie-experten.de DGAKI-Geschäftsstelle:info@dgaki.de

Quelle: Deutsche Gesellschaft für Allergologie und klinische Immunologie e.V. (DGAKI) 